\title{
Red de transporte de datos en formato RTCM, vía protocolo de Internet (Ntrip). Implementación en la región y proyección futura a través de SIRGAS
}

\author{
Norbertino Suárez Silva* \\ Roberto Pérez Rodino** \\ Ricardo Yelicich Peláez ${ }^{* * *}$
}

\begin{abstract}
The CORS (Continuously Operating Reference Station), use the GNSS (Global Positioning Satellite Systems) to determinate a high precision point by the observation of the available satellite constellations (GPS, GLONNAS, and the future Galileo and Compass/BeiDou). Using a technique called Differential Correction, is possible to eliminate almost completely the influence of the errors produced by the GNSS signals or induced errors. The Differential Correction methods (DGNSS) Post-Process or Real-Time are the most widely used methods. There are other solution type called PPP (Precise Point Positioning), and recently have been presented some progress on PPP in Real-Time: PPP-RTK by different satellites Geodetic Research Agency

The Differential Corrections may be obtained directly from a GNSS receiver or from CORS or from a network CORS, all connected to a Server-Caster via Internet.

The customers (rover receiver) use mobile phone technology (GSM, GPRS, EDGE, UMTS), a client software to access the Caster IP address, and Ntrip (Network Transport of RTCM data via Protocol Internet) to Differential Correction data for GNSS rover receiver.
\end{abstract}

* Tte. Cnel. Norbertino Suárez Silva, Inspector Técnico del Servicio Geográfico Militar (SGM). Delegado Nacional de Uruguay en SIRGAS, Montevideo, Uruguay, correos electrónicos: nsuarez@sgm.gub,uy, norbertinosuarez@gmail.com

** Ing. Agrim. Roberto Pérez Rodino, Profesor Agregado, Instituto de Agrimensura de la Facultad de Ingeniería de Uruguay (UDELAR), Presidente Grupo SIRGAS-RT, Montevideo, Uruguay, correo electrónico: rodino@fing.edu.uy

*** Ing. Agrim. Ricardo Yelicich Peláez, Instituto de Agrimensura de la Facultad de Ingeniería (UDELAR), Montevideo, Uruguay, correo electrónico: ryelicich@fing.edu.uy 
Ntrip is based on HTTP (HyperText Transfer Protocol) and has the ability to transmit any GNSS data through RTCM (Radio Technical Commission for Maritime Services) messages or raw data.

The customers (rover receiver) can choose different solutions: corrections from a single CORS, or corrections from a network CORS. There are differents network solutions: VRS (Virtual Reference Station), FKP (Correction Parameters planes), MAX (Master-Auxiliary), iMAX (individual Master-Auxiliary), etc.

During the SIRGAS (Geocentric Reference System for the Americas) meeting in Montevideo-Uruguay, in May 2008, the Working Group SIRGAS-RT was established in order to evaluate and promote Ntrip development and research. So far have been implemented Casters-Ntrip Servers in Argentina, Brazil, Venezuela and Uruguay, with significant results. Currently SIRGAS-RT is working on regional developments that including neighboring countries CORS networks. Argentina and Uruguay have installed some Experimental Casters-SIRGAS for research and regional technology evaluation.

Key words: GNSS, CORS, CORS networks, Ntrip, VRS, Caster, Real-Time, RTK, PPP-RTK, Positioning, Differentials Corrections.

\section{Resumen}

Las CORS (Estaciones de Referencia de Observación Continua), utilizan los GNSS (Sistemas Satelitales de Posicionamiento Global), para determinar con alta precisión una posición, mediante la observación de los satélites de las constelaciones disponibles (GPS, GLONNAS, próximamente Galileo y Compass/BeiDou).

Empleando la técnica llamada de Corrección Diferencial, es posible eliminar casi totalmente la influencia de los errores inducidos voluntariamente o producidos por la señal GNSS. El método DGNSS, tanto en modo Post Proceso como en Tiempo Real, son las modalidades más utilizadas. Actualmente están disponibles también soluciones PPP (Precise Point Positioning - Posicionamiento Puntual Preciso), a partir de desarrollos generados en varias agencias de investigación. Últimamente se han presentado ciertos avances sobre PPP (Posicionamiento de Punto Preciso), particularmente en tiempo real: PPP-RTK.

Las Correcciones Diferenciales pueden ser obtenidas directamente a partir de un receptor GNSS, de una CORS o de una red CORS, conectadas a un Servidor-Cáster a través de Internet.

El cliente o usuario móvil utilizando tecnología de telefonía móvil (GSM, GPRS, EDGE, UMTS) y un programa Cliente, puede acceder a la dirección IP del Cáster, a través de protocolo Ntrip (Red de Transporte de datos en formato RTCM a través Protocolo de Internet), a fin de obtener los datos de corrección diferencial para el receptor GNSS que está utilizando. 
Ntrip se basa en HTTP (HyperText Transfer Protocol - Protocolo de Transferencia de Hipertexto), siendo capaz de trasmitir cualquier tipo de datos GNSS a través de mensajes RTCM (Radio Technical Commission for Maritime Services) o datos en formato propietario (raw data). El usuario puede elegir entre una serie de productos, como por ejemplo correcciones de una sola CORS, o corrección de una red CORS. Existen diversas soluciones de red, como ser: VRS (Estación de Referencia Virtual), FKP (Parámetros de Corrección de Planos), MAX (Máster-Auxiliar), iMAX (individual Máster-Auxiliar), etc.

En el marco de la Reunión SIRGAS (Sistema de Referencia Geocéntrico para las Américas) llevada a cabo en Montevideo, Uruguay, en mayo de 2008, se crea el Grupo de Trabajo SIRGAS-RT, con la finalidad de evaluar e impulsar el desarrollo de la tecnología Ntrip en la órbita de los países participantes. Se han implementado hasta el momento Servidores-Cásters Ntrip en Argentina, Brasil, Venezuela y Uruguay, entre otros, con importantes resultados. Actualmente se está trabajando en desarrollos regionales que incluyan CORS de las redes existentes en países vecinos; Argentina y Uruguay ya cuentan con Cásters-SIRGAS experimentales, los que están en fase investigación y evaluación de potencialidades.

Palabras clave: GNSS, CORS, redes CORS, Ntrip, VRS, Caster, Tiempo-Real, RTK, PPP-RTK, Posicionamiento, Corrección Diferencial.

\section{Introducción}

La utilización de los Sistemas Globales de Navegación por Satélite (GNSS en su acrónimo inglés) ha crecido en forma exponencial en estos últimos años, presentando una fuerte demanda en aplicaciones como GIS, GIS Mobile, Agricultura de Precisión, Relevamientos Topográficos, Cartografía, Geodesia, Geofísica, entre otros, exigiendo además mejores precisiones en el posicionamiento geoespacial.

Las Estaciones de Referencia de Observación Continua (CORS), llamadas comúnmente estaciones permanentes, estaciones de referencia o estaciones fijas, son equipos que utilizan los GNSS, siendo capaces de determinar la posición con gran exactitud, observando satélites de las constelaciones GPS, GLONNAS, próximamente Galileo y Compass/BeiDou.

Las CORS son instrumentos geodésicos para posicionamiento global de alta precisión, que funcionan las 24 horas del día, los 365 días del año, observando continuamente las coordenadas geocéntricas del lugar.

Empleando la técnica llamada de corrección diferencial, que utiliza al mismo tiempo dos receptores satelitales, es posible eliminar casi totalmente la influencia de los errores inducidos voluntariamente o producidos por la señal GNSS, debido a su desplazamiento a través de la atmósfera, a los relojes de los satélites, inexactitud de las órbitas, etc. Al ubicar uno de los receptores en una estación con coordenadas conocidas (estación fija, por ejemplo una CORS), se pueden predecir (calcular) las 
distancias geométricas a los satélites y comparar con las distancias medidas del receptor a los satélites; esa diferencia es el error en la señal. Al aplicar la diferencia obtenida, a la distancia determinada con el receptor ubicado en la estación de coordenadas desconocidas (móvil), se corrige la misma, logrando altas precisiones en la determinación posicional. Esto es posible mediante la combinación de las observaciones realizadas simultáneamente por ambos equipos.

El método Diferencial, tanto en modo Post Proceso (DGNSS/PP) como en Tiempo Real (DGNSS/RTK), son las modalidades más utilizadas para lograr las precisiones requeridas. Actualmente están disponibles también las soluciones PPP (Precise Point Positioning - Posicionamiento Puntual Preciso), a partir de desarrollos generados en varias agencias de investigación. Particularmente el IGS (International GNSS Service) inició en el año 2007, un proyecto piloto sobre esta nueva forma de determinación de posiciones precisas. El PPP emplea datos de medición de fase no diferenciadas, provenientes de un solo receptor de tipo geodésico, incluyendo los datos de corrección de los errores en los relojes de los satélites y las órbitas precisas, junto a un esquema de modelamiento adicional de errores. Se obtienen con esta modalidad soluciones con exactitudes decimétricas y centimétricas. En este caso los "puntos fijos" pasan a ser directamente los satélites.

Atendiendo las necesidades de posicionamiento preciso en tiempo real y en virtud de los avances tecnológicos, a finales del año 2004, la BKG (Bundesamt für Kartographie und Geodäsie - Agencia Federal Alemana de Cartografía y Geodesia) desarrolló el protocolo Ntrip (Network Transport RTCM Internet Protocol), abriendo así una nueva posibilidad en cuanto a trasmisión de datos.

\section{El método Diferencial}

El método Diferencial consiste en calcular el vector tridimensional $(d X, d Y, d Z)$ entre dos puntos ocupados en forma simultánea por dos receptores GNSS, donde uno es considerado base y el otro como móvil. De esta forma, resolviendo el vector definido por ambos puntos y aplicando este vector a las coordenadas del punto base, podemos obtener las coordenadas del punto que ocupa el receptor móvil. La precisión en la posición relativa de estos puntos, es más alta que la posición relativa de los mismos puntos medidos en forma absoluta entre sí. Además, si se tienen las coordenadas absolutas precisas del punto base, las coordenadas absolutas del punto móvil serán también precisas; estas últimas solamente degradadas por la incertidumbre en el cálculo del vector. El equipo base puede ser un equipo propio del usuario (configuración típica de equipamiento topográfico), una CORS, o una red CORS.

La corrección diferencial se puede hacer de dos modalidades: Postproceso (DGNSS/PP) o en Tiempo Real (DGNSS/RTK). 


\section{Corrección Diferencial Post Proceso (DGNSS/PP)}

Las aplicaciones DGNSS/PP están limitadas a que la posición precisa de los puntos relevados con el receptor móvil, se logra con el cálculo posterior en gabinete. Esto hace que el método DGNSS/PP no sea aplicable en ciertos casos (replanteo, navegación precisa, etc.).

\section{Corrección Diferencial en Tiempo Real (DGNSS/RTK)}

El método de corrección DGNSS/RTK permite obtener las posiciones precisas del equipo móvil, directamente en el campo, en el momento del relevamiento. Para que esto sea posible es necesario contar con un enlace que transmita en tiempo real, los datos del receptor base al receptor móvil. Existen diversas opciones de enlace: radio-módem, satélite de comunicaciones, internet, entre otras. Generalmente todas tienen costo para el usuario, y están limitadas a una región de alcance máximo entre el receptor base y el receptor móvil.

El esquema es sencillo: el equipo base recibe la señal de los satélites GNSS, procesa las observables y envía los datos al equipo móvil, conjuntamente con las coordenadas de su estación, a través de algún tipo de enlace. El equipo móvil recibe los datos de la base al mismo tiempo que la señal de los satélites GNSS; los datos son procesados simultáneamente obteniéndose la posición diferencial del punto ocupado.

Los datos que envía la base al móvil pueden tener distintos formatos; en particular se pueden dividir en formatos propietario o formato RTCM-SC 104 (Radio Technical Commission for Maritime Services, Special Committee 104) en sus distintas versiones 2.x y 3.x. El formato de datos propietario es específico del fabricante de cada de equipo (marca comercial), y en general solo son usados por equipos de la misma marca comercial. Son conocidos como raw data.
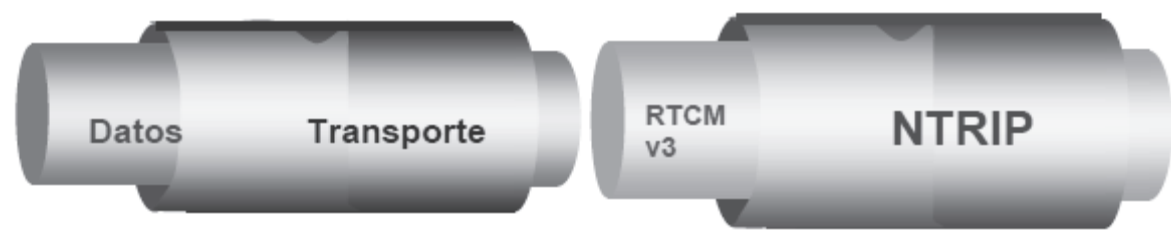

Figura 1. Flujo de datos, formato y protocolo a través de Ntrip. 
El formato RTCM tiene distintos tipos de mensajes. En la versión 2.x los mensajes tipo 1 y 3 , envían solamente la corrección diferencial simple de código y los parámetros de la estación fija respectivamente; los tipo 18 y 19 envían los datos de la fase y código en L1 y L2 de la estación fija, así como los tipo 20 y tipo 21 envían las correcciones RTK para L1 y L2, etc. Finalmente en la versión 3.x los datos están más compactados y otorgan muchas más alternativas de tipos de mensajes a enviar; optimiza el ancho de banda, y tiene además un menú más amplio de datos posibles.

Evidentemente según el mensaje que se necesite y dependiendo del tipo de receptor utilizado se conseguirá distinto volumen de tráfico de datos y diferentes precisiones podrán ser alcanzadas (submétricas, centimétricas).

\section{Trasmisión de datos al móvil}

En forma general las posibilidades pueden ser resumidas en: vía beacon, vía radiomódem, vía satélite de comunicaciones, vía conmutación telefónica y vía Internet.

El beacon es una emisora que trasmite las señales en las bandas de radio con alcances del orden de los 400-500km. Por lo general se necesita un abono a la señal y comúnmente ofrecen solamente correcciones con precisiones métricas o submétricas.

El radio-módem es la solución del tipo particular (mono-usuario), empleada generalmente en trabajos topográficos. El usuario tiene dos equipos que los enlaza entre sí por radio-módems, los que transmiten en general en UHF. Por lo tanto su radio de acción es limitado (se reduce a pocos $\mathrm{km}$ ), obteniéndose precisiones centimétricas.

Mediante el uso de satélites de comunicaciones se han implementado servicios de corrección de las señales que brindan los GNSS, al receptor de los usuarios, denominados SBAS (Satellite Based Augmentation System - Sistema de Aumentación Basado en Satélites). Podemos citar varios servicios de esta naturaleza: el WASS (Wide Area Augmentation System) gestionado por el Departamento de Defensa de los Estados Unidos de América, el EGNOS (European Geostationary Navigation Overlay Service) gestionado por la Agencia Espacial Europea, el MSAS (Multi-Functional Satellite Augmentation System) gestionado por Japón, y el GAGAN (GPS and GEO Augmented Navigation) planificado por India. Existe otra implementación con fines comerciales llamada OmniSTAR, que brinda un servicio corrección diferencial, para lo cual se debe contratar la señal. Estos sistemas proporcionan los datos de correcciones de distintas estaciones. Según la región, el tipo de servicio y el alcance del contrato, se puede acceder a distintas señales, con diferentes precisiones y costos.

La conmutación telefónica es el enlace punto a punto a través de una llamada de datos. Esta solución es individual y sus costos de comunicaciones en general son muy elevados. 
Finalmente tenemos la distribución de datos por internet. Como se ha mencionado, a finales del año 2004, el BKG desarrolló el protocolo Ntrip, abriendo así una nueva modalidad en cuanto a trasmisión de datos.

Ntrip da la posibilidad de tener acceso y mejorar el flujo de datos GNSS de las redes CORS, a una variedad de clientes (usuarios), a través de una técnica de comunicación definida. Esta solución depende de la trasmisión de datos vía telefonía móvil. Utiliza las modalidades GPRS, EDGE, 3G, UTMS, etc., destacándose el menor costo en la trasmisión de datos, frente a otras formas de comunicaciones.

La distribución de datos GNSS a través de Internet utilizando esta modalidad, es cada vez más común, debido a su fácil instalación y acceso. El desarrollo de los sistemas de acceso a Internet móvil a través de GPRS (General Packet Radio Service) y GSM (Global System for Mobile Communication), proporciona un método rápido y seguro para la distribución de datos GNSS o transmisión de correcciones DGPS/RTK, a un receptor ubicado en aquellas zonas que tienen cobertura de telefonía móvil.

Las precisiones que se pueden alcanzar son centimétricas o decimétricas, dependiendo de la distancia a la CORS en soluciones punto a punto, y de las características del receptor móvil. Si se dispone de una red CORS, se pueden generar soluciones de red con los algoritmos del servidor, del tipo: VRS (Virtual Reference Station), FKP (Flächen-Korrectur-Parameter), MAX (Master Auxiliary Corrections) o iMax (Individual Master Auxiliary Corrections).

\section{Descripción de Ntrip}

El Ntrip se refiere a una Red de Transporte de datos en formato RTCM (Radio Technical Commission for Maritime Services), a través del Protocolo de Internet.

Este protocolo intenta revertir las desventajas presentes en mediciones DGNSS/RTK, particularmente la limitación de distancia entre la ubicación de la estación fija y el móvil, debido a problemas de comunicaciones a través de sus radio-módems.

Sus características principales son:

- Está basado en el protocolo de hipertexto HTTP/1.1 (Hypertext Transfer Protocol); actúa en la capa más alta del TCP/IP (Transmission Control Protocol / Internet Protocol).

- Utiliza el popular estándar HTTP streaming, por medio de IP (Protocolo de Internet). Luego en la versión 2 de Ntrip también usa el RTP (Real-Time Transport Protocol), que esta soportado por el protocolo UDP (User Datagram Protocol).

- Es capaz de atravesar "firewalls" y "proxies" que permiten el paso de HTTP estándar. 
- La finalidad es dar acceso y mejorar el flujo de datos de las CORS, a una variedad de clientes (usuarios) a través de internet.

- Los datos enviados pueden ser en formatos RTCM, "raw data" y otros tipos de datos como ser corrección de relojes, efemérides, etc.

El streaming (flujo continuo, sin interrupción), surge con la finalidad de conectar el flujo de datos que proporciona una CORS, con un móvil en el campo. Es importante que varios usuarios puedan conectarse a una misma CORS en forma simultánea, o que varias CORS estén disponibles para varios usuarios, etc. Las Redes CORS brindan estas posibilidades.

Este método emplea una serie de CORS que recolectan datos de los GNSS en forma continua. Los datos son enviados por Internet a un servidor central (Cáster), donde se calculan los parámetros de corrección para ser enviados al usuario a través de Internet. El usuario debe contar con una antena y un receptor GNSS capaz de recibir mensajes RTCM y conexión a Internet. Esta conexión puede ser mediante módem inalámbrico, una tarjeta SIM (Subscriber Identity Module - Módulo de Identificación de Abonado) incorporada a la antena/receptor o colectora de datos, o una conexión bluetooth por intermedio de un teléfono móvil. El usuario puede elegir entre una serie de productos, como por ejemplo correcciones de una sola CORS, o corrección de una red CORS. Al mismo tiempo, existen diversas soluciones de red, entre ellas, VRS, FKP, MAX, iMAX, etc.

\section{Arquitectura de Ntrip}

NtripSource: el hardware involucrado en este segmento son las CORS generadoras de las correcciones diferenciales para su aplicación a las mediciones GNSS, que incluyen un receptor GNSS y una antena geodésica que observa continuamente las constelaciones de satélites.

NtripServer: Este componente necesita una conexión con los datos generados por NtripSource además de un computador con conexión a Internet o intranet capaz de ejecutar el software para la transmisión de correcciones al NtripCáster.

NtripClient/NtripUser: El requerimiento básico para recibir correcciones diferenciales mediante el uso de Ntrip es que el receptor GNSS tenga la opción de recibir correcciones DGNSS/RTK, u otro tipo de correcciones, p.e. SSR (System Space Representation), con motor de cálculo. En su defecto se puede utilizar otro dispositivo intermediario (PC, Palm, Tablet, iPad, Smartphone, etc.) con el software adecuado. Complementariamente se necesitan dispositivos de comunicación como teléfonos móviles (celulares) con la capacidad de recibir y enviar datos vía Internet. Actualmente la mayoría de los receptores GNSS geodésicos cuentan con esta posibilidad, incluyendo el software para Ntrip Cliente, y zócalo para módem GPRS/3G. 


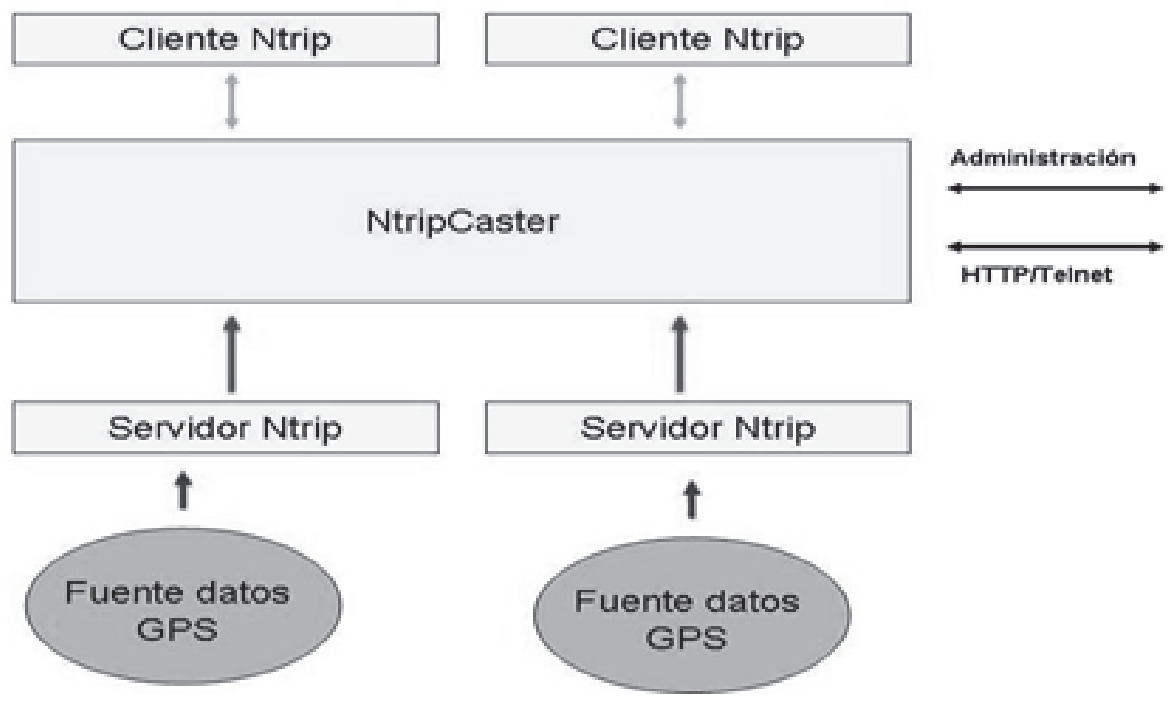

Figura 2. Flujo de datos NTRIP.

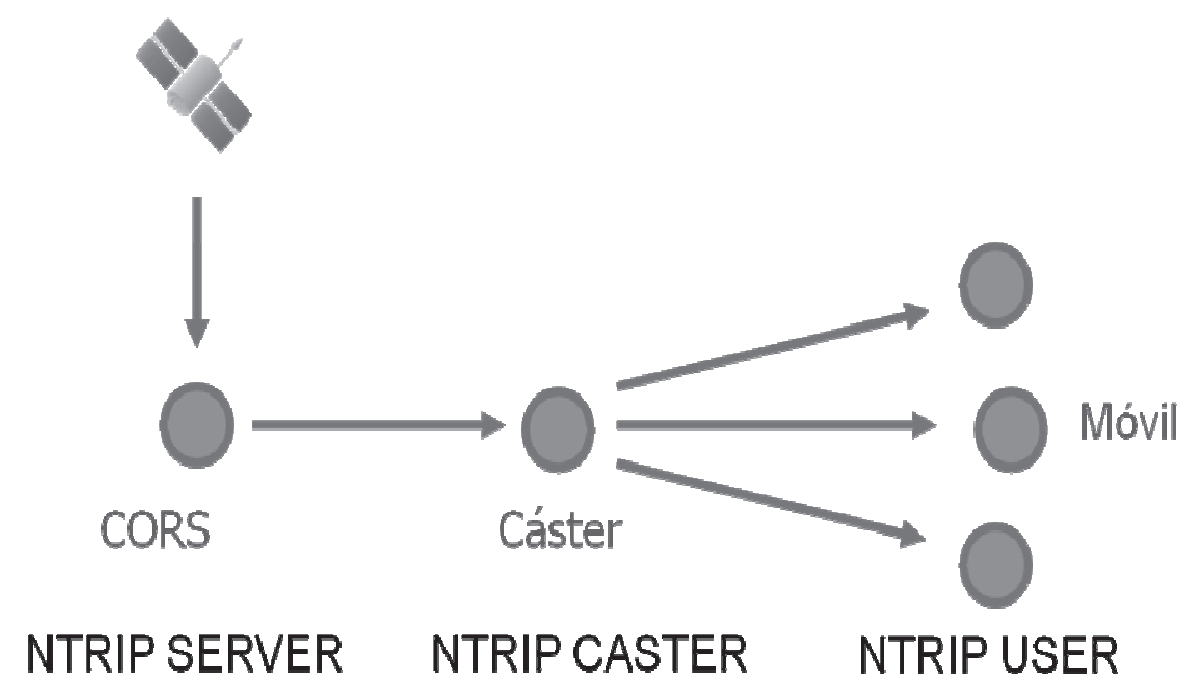

Figura 3. Arquitectura Ntrip.

\section{Soluciones de Red}

Las soluciones de red se utilizan para modelar y corregir aquellos errores dependientes de la distancia que reducen las precisiones en el cálculo de la posición del 
usuario. Las fuentes de error más significativas son el error ionosférico, el error troposférico, el error en los parámetros orbitales y el error en los parámetros de los relojes.

Uno de los primeros productos de soluciones de red fueron: la Estación de Referencia Virtual, VRS por sus siglas en inglés (Virtual Reference Station) y los Parámetros de Corrección por Planos, FKP por sus siglas en alemán (Flächen Korrektur Parameter). Luego aparecieron otras soluciones, como el Concepto Master-Auxiliar (MAX) o el i-MAX (Individual Master-Auxiliary Concept), entre otras.

\section{VRS (Estación de Referencia Virtual)}

El concepto de la Estación de Referencia Virtual es una técnica de creación de datos GNSS para una CORS virtual, inexistente materialmente, con el fin de mejorar los resultados alcanzados con el posicionamiento RTK convencional, proporcionando correcciones RTK basadas en una red Estaciones de Referencia.

La red CORS vinculada a un Centro de Control, genera una base de datos de correcciones de área, regionales. Esta es utilizada para crear una VRS, situada a pocos metros de del receptor móvil. El receptor móvil interpreta y utiliza los datos de la VRS como si provinieran de una CORS real.

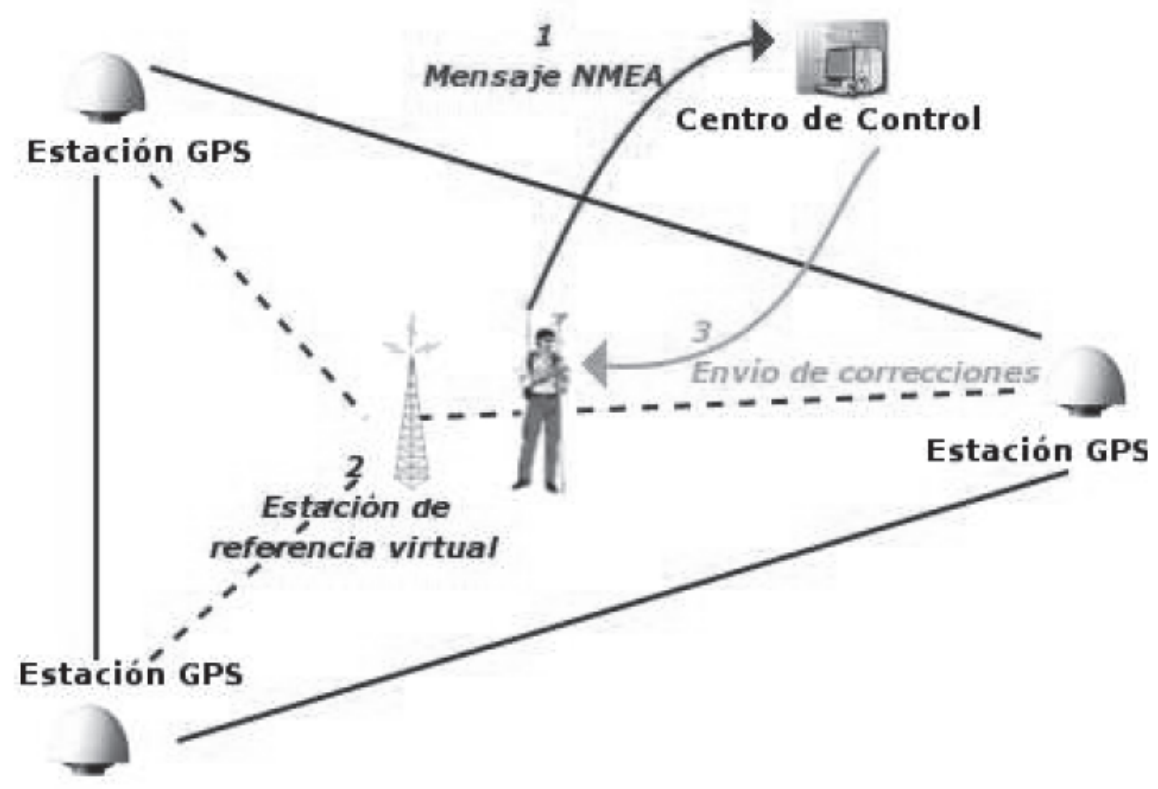

Figura 4. Esquema de conectividad en arquitectura VRS. 
La implementación de la técnica VRS necesita de al menos tres CORS conectadas el servidor de red. El móvil debe ser capaz de mantener una comunicación de ida y vuelta, y ha de estar ubicado dentro de alguna de las "celdas" definidas por un grupo de estaciones. El usuario se conecta al servicio de red VRS vía acceso a Internet móvil y una vez que se ha autentificado correctamente, el receptor móvil envía su posición navegada en formato NMEA (National Marine Electronics Association) al Centro de Control. Éste acepta la posición como la ubicación de una nueva CORS, calculando correcciones relativas a la VRS creada recientemente, y las envía al receptor móvil en formato RTCM o propietario.

\section{FKP (Parámetros de Corrección de Planos)}

El FKP utiliza superficies polinomiales (normalmente planos) que modelan los errores en las observaciones, en función de la distancia a la CORS. Para generar todas las correcciones necesarias, cada CORS debe generar un plano para cada señal (frecuencia) de la constelación y para cada satélite visible.

La técnica emplea la información de las CORS con el fin de derivar parámetros lineales que describen el efecto de los errores atmosféricos y de la órbita de satélites. Estos parámetros se diseminan a los usuarios como gradientes a ser utilizados para la interpolación de los errores de la red, a su posición real. Las variaciones espaciales de los errores se corrigen por "planos" de interpolación de primer grado.



Figura 5. Planos polinomiales del FKP que modelan los errores de las observaciones. 
El eje $x$ define la longitud, el eje $y$ la latitud, mientras que el eje $z$ corresponde a la magnitud de la corrección que puede ser para la fase o para la pseudodistancia. Cada "plano" define un área en que los parámetros correspondientes son válidos. El usuario que opera dentro de la red recibe los parámetros de corrección de la CORS más cercana.

Su principal ventaja es que la corrección no necesita de un canal bidireccional de datos como en VRS y se puede emitir en broadcast con un transmisor de radio, porque es el receptor el que se encarga de corregir su posición con los parámetros facilitados. La desventaja principal de esta técnica se refiere a que el móvil se debe encargar de calcular la corrección para su posición, con el aumento en la complejidad y potencia de cálculo necesario en éste.

\section{MAC (Concepto Master-Auxiliar)}

El concepto relativamente nuevo de Master- Auxiliar (MAC), fue diseñado para transmitir los datos de corrección, de una red CORS al móvil.

Es fundamentalmente la evolución hacia un estándar del FKP, por lo tanto posee las mismas ventajas que el FKP, esto es, emisión unidireccional de datos y empleo de CORS reales, no virtuales. Además el formato de las correcciones permite un menor ancho de banda en las transmisiones.

El concepto MAC se ha estandarizado en el nuevo formato RTCM 3.1. Utiliza una forma altamente compacta (menor número de bits), mediante la representación de datos de observación con ambigüedades niveladas, así como las diferencias de corrección de los datos de dispersión y no dispersivo para cada par receptor-satélite.

Con el fin de reducir el volumen de datos a transmitir por una red (información completa de antena, coordenadas y de corrección) sólo se envían datos para una sola CORS (la estación maestra). Para el resto de las estaciones de la red o sub-red (las estaciones auxiliares), junto con las correcciones convencionales se emitirán otro conjunto de correcciones que representan las diferencias relativas entre la estación maestra y las estaciones auxiliares. Estas correcciones son las que introducen el concepto de red.

Se dice que dos CORS están en un nivel de ambigüedad común si se han eliminado o ajustado las ambigüedades enteras para cada rango de fase (par receptorsatélite), de forma tal que las ambigüedades enteras se cancelan frente as dobles diferencias (que implica dos receptores y dos satélites) surgidas durante el procesamiento. La determinación de las ambigüedades enteras entre las CORS, es la función fundamental de los programas de procesamiento de la red. La reducción de las observaciones originales de las CORS sin procesar en el mismo nivel de ambigüedad, no altera las propiedades generales de las observaciones de fase portadora (efectos de la ionosfera y la troposfera, las variaciones del centro de fase de antena, etc.), ya que se han introducido sólo números enteros. Una aplicación que acceda a 
las observaciones de ambigüedad niveladas de una sola CORS, no verá ninguna diferencia ya que los requisitos de modelado dentro de la aplicación son idénticos. Sin embargo, cuando una aplicación utiliza las observaciones de más de una CORS, ya no tienen que dar cuenta de las ambigüedades de enteros entre las CORS en el mismo nivel de ambigüedad. Esto significa que un receptor móvil que reciba y utilice observaciones de ambigüedad niveladas de más de una CORS puede cambiar de una CORS a otra sin re-inicialización de su filtro.

Dividir las correcciones en componentes dispersivas y no dispersivas reduce aún más el ancho de banda requerido, ya que la troposfera y los errores de órbita son conocidos por cambiar sólo lentamente con el tiempo, por lo que la velocidad de datos no necesita ser tan alta como para el error dispersivo. Como la estación maestra se utiliza simplemente para fines de transmisión de datos y no juega ningún rol especial en el cálculo de las correcciones, no necesariamente debe ser la CORS más cercana al móvil, la utilizada. El usuario puede emplear estos mensajes ya sea en el modo broadcast (comunicaciones de un solo sentido) o en modo automático (comunicaciones de ida y vuelta). El modo de diseminación de la estación maestra está predeterminado por el operador de red, mientras que será la estación más cercana al móvil la que dispondrá del modo automático.

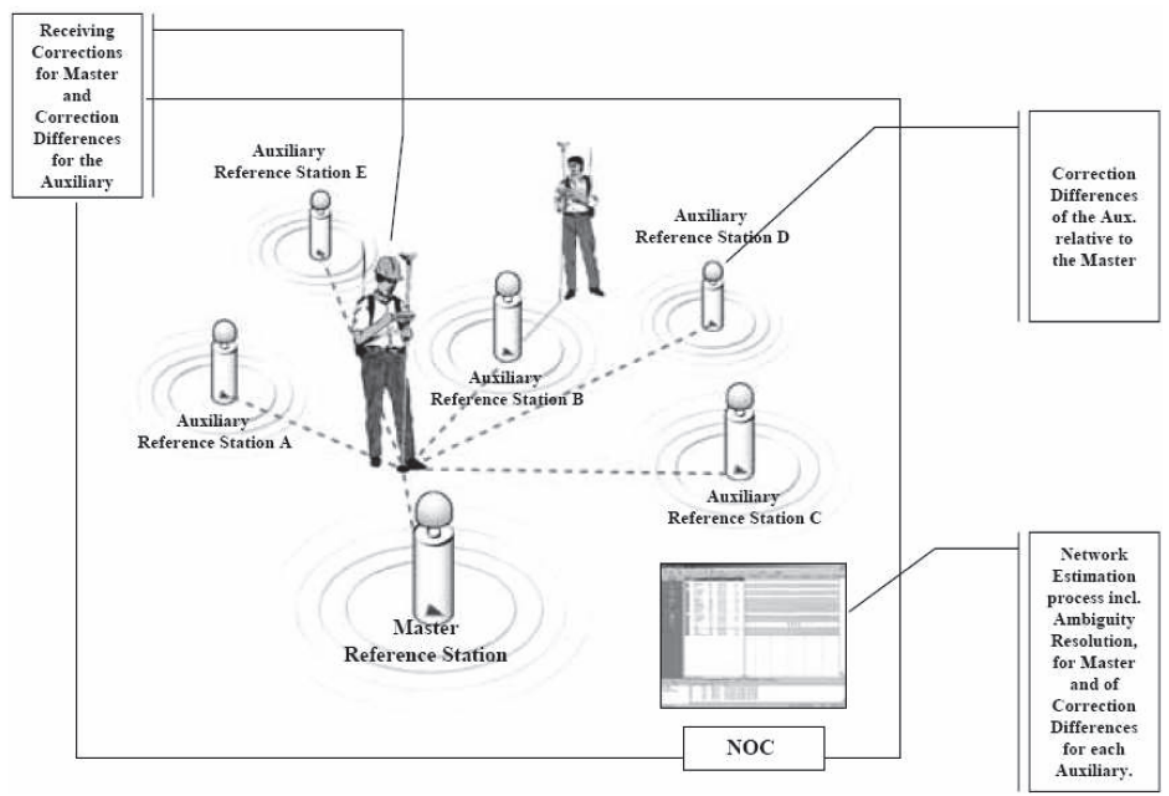

Figura 6. Arquitectura Máster-Auxiliary. 
Existen dos formas de implementar este concepto, MAX e i-MAX. La primera Master Auxiliary Corrections contiene las correcciones de red MAX especificadas anteriormente. La interpolación de las correcciones de red se realiza en el móvil, usando la información completa de la red; pero solo está disponible para móviles que soportan estos mensajes. Las correcciones MAX están disponibles en ambos modos de comunicación: en un solo sentido y en ida y vuelta. Es importante destacar que si las correcciones de red no están disponibles (número insuficiente de ambigüedades fijas para pares receptor-satélite), entonces no se enviarán las correcciones.

La segunda, individualised-Master Auxiliary Corrections, se creó para que el modelo pueda ser utilizado por receptores existentes que no pueden interpretar mensaje MAX. Este esquema requiere de comunicaciones de ida y vuelta para que el operador de la red pueda elegir las celdas apropiadas e interpolar las correcciones de red para la posición del móvil. El método es similar al MAX, pero las correcciones de red se realizan en el servidor y son aplicadas a la estación maestra.

\section{El PPP-RTK (Posicionamiento de Punto Preciso)}

El concepto de PPP (Posicionamiento de Punto Preciso) hace referencia al posicionamiento absoluto.

Como se sabe los sistemas GNSS dan en forma nativa el posicionamiento absoluto en tiempo real, con el inconveniente de que la precisión puede alcanzar varios metros. Esta degradación de la precisión depende de los errores de las órbitas broadcast, los errores de los relojes, los errores atmosféricos (ionosféricos y troposféricos) y otros efectos no modelados en el método de posicionamiento absoluto simple.

Desde hace algunos años se empezaron a implementar aplicaciones Post Proceso llamadas PPP. Estas hacían uso fundamentalmente de las efemérides precisas y la corrección de relojes, y en caso de equipos de doble frecuencia se podía modelar la ionosfera, y utilizar distintos modelos troposféricos, en caso de que estuvieran disponibles.

Uno de los servicios que brinda el IGS es la generación de datos de órbitas precisas y de corrección de relojes, a partir de los datos capturados por las estaciones IGS. Estos productos son generados por distintos centros de procesamiento asociados y las soluciones son acordadas en una única solución IGS combinada. El IGS pone a disposición diferentes productos: las órbitas y relojes ultra-rápidos (disponibles a priori como pronóstico), las soluciones rápidas (al día siguiente de las observaciones) y las soluciones finales o precisas (aproximadamente a los 15 días).

Con las actuales posibilidades de conectividad a través de Internet que presentan los servicios de datos móviles, se empieza a vislumbrar en la modalidad PPP, particularmente en tiempo real (PPP-RTK), una nueva alternativa para brindar 
correcciones diferenciales en tiempo real. Varios centros de procesamiento de datos han empezado a generar flujos de datos con correcciones de relojes y efemérides a través de "streaming", usando el protocolo Ntrip. Ya no son datos estáticos (archivos para bajar); las correcciones se envían en formato RTCM 3.x, a través de Internet.

Cambia también el concepto de datos a transmitir. No se trasmiten las efemérides precisas sino que se envían las correcciones de las efemérides broadcast, etc., con la finalidad de que los paquetes de datos sean extremadamente cortos. Los mensajes incluyen además los ajustes al Sistema de Referencia con el que se desee trabajar. Estas correcciones denominadas SSR (System Space Representation) representan los errores y eventos del sistema en el espacio. Con el SSR se pueden modelar los errores existentes en GNSS: el error de reloj en el satélite, el error en las órbitas, error de demora en el sistema, error por cambios en la geometría de antena del satélite, errores ionosféricos, troposféricos, de geometría de antena receptora, la demora en el receptor, los Bias entre Constelaciones (CB) de GPS, GLONASS, GALILEO, etc.

Para hacer posible el PPP-RTK es necesario contar con los datos de correcciones, con los datos observados por el receptor utilizado para obtener PPP, la trasmisión de las efemérides brodcast y el software Cliente, que tenga la posibilidad de procesar y generar la solución buscada. Entre las herramientas desarrolladas hasta el momento se pueden citar: el BNC (BKG Ntrip Client), el BNC modificado por el CNES y el RTKLib. Son de código abierto y generalmente corren en plataforma Linux, existiendo algunas compilaciones para Windows. Las precisiones logradas se encuentran en el entorno de $10-15 \mathrm{~cm}$ en posición absoluta, con solución generalmente flotante, con determinado tiempo de convergencia. Hay estudios para fijar ambigüedades enteras; por ejemplo el desarrollado por el organismo gubernamental francés CNES (Centre National D'Etudes Spatiale) a través del Proyecto PPPWIZARD (Precise Point Positioning - With Integer And Zero Difference), en los cuales se llega a precisiones de unos pocos centímetros, con tiempos de 1 hora de convergencia.

En el Simposio PPP-RTK \& Open Standars, llevado a cabo en marzo de 2013 en Frankfurt Alemania, se presentaron 49 trabajos sobre esta temática (http://igs.bkg.bund.de/Ntrip/symp), lo que deja entrever el nivel de desarrollo académico en esta área del conocimiento. Se puede inferir entonces con propiedad, que los tiempos de resolución de ambigüedades y estabilización de soluciones, van reducirse drásticamente; las precisiones van a mejorar y la accesibilidad a Internet va a ser cada vez más universal. Seguramente aparecerán soluciones comerciales que incluyan el receptor GNSS, el módem de telefonía móvil y el software de PPP, en una sola unidad, posibilitando que un usuario, con un solo equipo, logre altas precisiones de posicionamiento absoluto en el Sistema de Referencia elegido. 


\section{Ntrip en SIRGAS}

En la Reunión del Sistema de Referencia Geocéntrico de las Américas (SIRGAS), realizada en mayo de 2008 en Montevideo, se resolvió: "Establecer un proyecto piloto denominado SIRGAS en tiempo Real (SIRGAS-RT), el cual tendrá como objetivo investigar los fundamentos y aplicaciones asociadas a la distribución, en la región SIRGAS, de observaciones y correcciones a las mediciones GNSS en tiempo real, mediante Ntrip o cualquier otro medio de largo alcance" (Boletín SIRGAS N ${ }^{\circ}$ 13, agosto, 2008); creando así el Grupo de Trabajo denominado SIRGAS-RT.

Desde entonces, Argentina, Brasil, Venezuela y Uruguay comenzaron a trabajar en la implementación de servicios Ntrip. Los primeros resultados de los estudios que se han realizado en este marco, fueron presentados en la Reunión Científica de la Asociación Internacional de Geodesia (IAG) en Buenos Aires, en setiembre de 2009, así como en las siguientes ediciones anuales de la Reunión SIRGAS.

En el Uruguay se inauguró oficialmente en el año 2007, la Red Geodésica Nacional Activa de la República Oriental del Uruguay (REGNA-ROU), desarrollada y administrada por el Servicio Geográfico Militar (SGM). Esta red cuenta actualmente con nueve Estaciones de Referencia de Observación Continua (CORS) activas. Todas las CORS están conectadas a través de Internet móvil GPRS/3G y sus datos son publicados en un servidor central (Cáster) ubicado en el SGM. Las Estaciones de la REGNA-ROU, integran la Red del Sistema de Referencia Geocéntrico para las Américas-Continuo (SIRGAS-CON); por tanto las observaciones de cada una de ellas es procesada conjuntamente con las demás Estaciones del continente, obteniendo una solución semanal ajustada a SIRGAS (Sistema de Referencia Geocéntrico para las Américas), formando parte además del Marco de Referencia Oficial del Uruguay denominado SIRGAS-ROU98 (Época 1995.4). El servicio es público, gratuito y sin restricciones para todos los usuarios y se accede a los datos de las estaciones a través de 26 mountpoints generados hasta el momento, con distintos tipos de soluciones, incluyendo soluciones de red (VRS, FKP, MAX e i-MAX). Se prevé la densificación de la Red para los próximos años, pretendiendo alcanzar un total de 22 estaciones homogéneamente distribuidas en el territorio, con una separación aproximada de $70 \mathrm{~km}$.

Al mismo tiempo, en el Instituto de Agrimensura de la Facultad de Ingeniería de la Udelar, se puso en marcha un Cáster experimental denominado "Cáster Escuela Ntrip" para colaborar con el proyecto SIRGAS-RT. Este Cáster de uso académico tiene por finalidad realizar investigaciones, pruebas y evaluaciones, pudiendo incorporar estaciones de la red existente, así como nuevas estaciones o estaciones de países de la región.

Brasil cuenta con la Red Brasileña de Monitoreo Continuo de los Sistemas GNSS (RBMC). En el año 2008 se implementó el servicio RBMC-IP, para posicionamiento en tiempo real a partir de la RBMC, para usuarios que utilizan el método 
RTK (cinemático en tiempo real) o DGPS (Diferencial para Sistema de Posicionamiento Global) en sus levantamientos. Los datos de corrección diferencial u otros tipos de datos GNSS para usuarios (móviles o estacionarios) se distribuyen vía Ntrip, a través de Internet. Permite la conexión simultánea de PCs, Laptops y PDAs que tienen acceso a Internet inalámbrica, empleando módems GPRS/3G.

En Argentina, la Red Argentina de Monitoreo Satelital Continuo (RAMSAC) a través del servicio RAMSAC-Ntrip cumple con los mismos objetivos de los servicios disponibles en Uruguay y Brasil, incorporando algunas CORS vecinas de la REGNA-ROU. Ha puesto en funcionamiento además un servicio denominado "Cáster SIRGAS Experimental", cuya finalidad es la publicación de datos GNSS en tiempo real utilizando el protocolo Ntrip. El Cáster se encuentra alojado en el Laboratorio del Grupo de Geodesia Satelital de Rosario, de la Universidad Nacional de Rosario, Argentina. Los usuarios interesados en acceder a los datos pueden hacerlo en forma libre y gratuita, luego de completar un formulario de registro, aceptar las condiciones de uso y recibir un nombre y una contraseña que les permita el acceso a los datos de su interés.

En Venezuela se estuvo trabajando desde el año 2009 en la implementación de un servicio Ntrip. Las pruebas realizadas por el Centro de Procesamiento y Análisis GNSS SIRGAS de la Universidad del Zulia (CPAGS-LUZ), han estado centradas en darle continuidad a los ensayos desarrollados desde 2009, cuando se evaluó el método midiendo sobre líneas de base cortas, medias y largas, desde una CORS en particular. La limitante que representa el acceso a Internet ha sido superada en los últimos años, persistiendo aún la escasez de estaciones activas generadoras y transmisoras de correcciones diferenciales. En cuanto a la implementación de PPP-RTK a través de Ntrip, el CPAGS-LUZ ha realizado algunas pruebas piloto, utilizando en primera instancia el software BNC v2.4, ofrecido por el BKG, el cual como lo hemos visto, además de permitir el acceso a correcciones de órbitas y parámetros de reloj de los satélites para lograr la estimación precisa en forma absoluta, también posibilita la aplicación de parámetros de transformación para la expresión de las coordenadas a los marcos SIRGAS95 y SIRGAS2000.

Con el correr de los años se fueron sumando algunas iniciativas en países como Ecuador, Chile y Perú, pero en estos casos han prevalecido sobre todo desarrollos privados, por lo que no cuentan aún con Cásters nacionales que publiquen los datos de todas las CORS de su territorio.

En Puerto Rico, se ha implementado un Cáster, cuya principal función es alertar sobre terremotos y tsunamis.

El Grupo SIRGAS-RT dispone actualmente de dos licencias del software NtripCaster Professional, desarrollado por la Bundesamt für Kartographie und Geodäsie (BKG), de Alemania y donado por esta Agencia a la comunidad SIRGAS. Estas licencias están siendo utilizadas por el grupo LEPODA-G (Laboratorio Expe- 
rimental de Procesamiento de Datos GNSS) del Instituto de Agrimensura de la Facultad de Ingeniería de Uruguay (Udelar) y por el Laboratorio del Grupo de Geodesia Satelital de Rosario de la Facultad de Ciencias Exactas, Ingeniería y Agrimensura, Universidad Nacional de Rosario, Argentina. La finalidad es apoyar las iniciativas "Cáster Escuela Ntrip" y "Cáster SIRGAS Experimental" respectivamente de ambas instituciones, las que fueron descritas anteriormente. LEPODA-G cuenta además con una licencia temporal del software comercial SpiderNet de Leica, la que es empleada en el Proyecto.

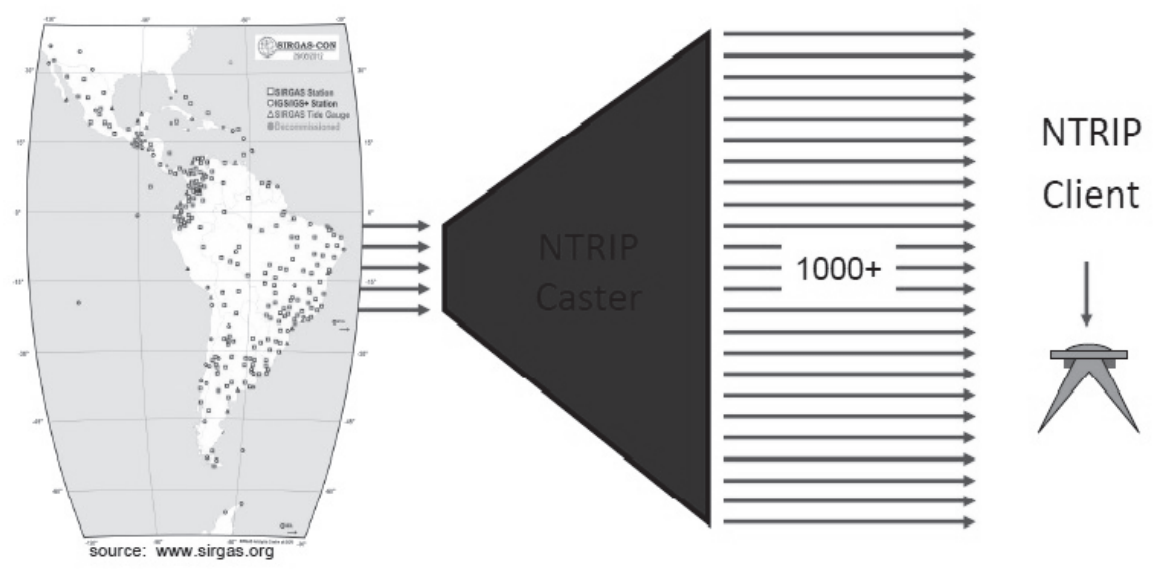

Figura 7. Concepto de implementación Cáster-Ntrip SIRGAS.

En la reunión SIRGAS 2012, en la ciudad de Concepción, Chile, se expusieron nueve trabajos asociados al posicionamiento en tiempo real, de un total de 64 trabajos presentados, lo cual demuestra la importancia que está teniendo la temática dentro de SIRGAS. Los trabajos de aplicación hicieron referencia, por ejemplo, a la utilización de un Sistema Terrestre de Aumentación GBAS para la fase de vuelo, aproximación y aterrizaje de aviones en Colombia, o el uso de diversas técnicas de posicionamiento RTK (Ntrip, Omnistar y PPP-RTK) frente al método RTK convencional (UHF), como soporte a la georreferenciación durante el proceso de exploración petrolera en Venezuela. Algunos trabajos estaban relacionados con la implementación de soluciones RTK mono-estación y VRS en Perú y Uruguay. Se realizaron dos presentaciones sobre experiencias PPP-RTK utilizando el software BNC; una en Rosario, Argentina y otra en Montevideo, Uruguay. De la misma manera, se realizaron exposiciones sobre las precisiones obtenidas con la técnica RTK-Ntrip en Ecuador y en Argentina; en este último caso se sumaron los resulta- 
dos generados al utilizar únicamente el observable de código en experiencias DGPS y PPP.

Por el momento, se publican datos en tiempo real de algunas CORS de la región, así como también efemérides y correcciones a los relojes, de manera que pueden desarrollarse proyectos apoyados en posicionamiento relativo o PPP, ambos en tiempo real.

\section{Datos de Cásters Nacionales}

RBMC-IP. IBGE (Brasil)

Dirección IP: 186.228.51.52

Puerto: 2101

Software utilizado: Ntrip Caster 1.5.8/1.0

Cantidad de estaciones: 45

Formato: RTCM 3.0

Otros productos: Correcciones de órbitas y relojes (SIRGAS200001 (GPS+GLO), SIRGAS2000.02 (GPS), IGS03) y Efemérides (RTCM3EPH)

Datos de acceso: Solicitud de registro de usuarios en http://www.ibge.gov.br/ espanhol/geociencias/geodesia/rbmc/Ntrip/cadastro.php

Marco de Referencia: SIRGAS2000

Época: 2000.4

URL: <http://www.ibge.gov.br/espanhol/geociencias/geodesia/rbmc/Ntrip/>

REGNA-ROU. SGM (Uruguay)

Dirección IP: 200.40.69.58

Puerto: 8081

Software utilizado: GNSS Spider/2.0

Cantidad de estaciones: 9

Formato: RTCM 2.3-3.0

Otros productos: Correcciones de red (VRS, FKP, MAX, i-MAX)

Datos de acceso: No requiere registro de usuario.

Marco de Referencia: SIRGAS-ROU98

Época: 1995.4

URL: <http://www.sgm.gub.uy/geoportal/index.php/estaciones-referencia/srv-tr/>

RAMASAC-Ntrip. IGN (Argentina)

Dirección IP: 190.220.8.208

Puerto: 2101

Software utilizado: Ntrip Cáster 2.0.14/2.0

Cantidad de estaciones: 22 
Formato: RTCM 2.3-3.0

Datos de acceso: Usuario: ramsac-Ntrip; Contraseña: ign_argentina_2010

Marco de Referencia: POSGAR07

Época: 2006.6

URL: <http://www.ign.gob.ar/AreaProfesional/Geodesia/RamsacNtrip/>

PRSN. UPR (Puerto Rico)

Dirección IP: gps1.uprm.edu/136.145.162.224

Puerto: 2101

Software utilizado: Ntrip Caster Professional 2.0.15/2.0

Cantidad de estaciones: 13 (de Puerto Rico, Costa Rica, Nicaragua, Jamaica, Curacao, St Thomas)

Otros productos: Correcciones de órbitas y relojes (CLK11 BRDC_APC_ITRF) y Efemérides (EPH Assisted-GNSS)

Formato: RTCM 3.0

URL: $<$ http://prsn.uprm.edu/ $>$

IGN (Perú)

Dirección IP: 190.12.71.75

Puerto: 2101

URL: <http://ign.gob.pe/>

(no operativo)

\section{Cásters de SIRGAS}

UNR (Argentina)

Dirección IP: 200.3.123.65

Puerto: 2101

Software utilizado: Ntrip Caster Professional 2.0.15/2.0

Cantidad de estaciones: 7

Otros productos: Correcciones de órbitas y relojes: SIRGAS2000, SIRGAS95, IGS03. Efemérides: RTCM3EPH

Formato: RTCM 3.0 - 2.3

Datos de acceso: Solicitud de registro de usuarios es http://www.fceia.unr.edu.ar/gps/caster/caster-user.php/

URL: <http://www.fceia.unr.edu.ar/gps/caster/>

UDELAR I (Uruguay)

Dirección IP: 164.73 .34 .31

Puerto: 8081 
Software utilizado: Ntrip Caster Professional 2.0.15/2.0

Cantidad de estaciones: 1 (variable) de acuerdo a los análisis que se estén haciendo)

Datos de acceso: sin registro

URL: (no operativo; solo Escuela)

\section{UDELAR II (Uruguay)}

Dirección IP: 164.73.34.17

Puerto: 8081

Software utilizado: Spider - Leica

Cantidad de estaciones: 6 (variable) de acuerdo a los análisis que se estén haciendo) Entrega soluciones de Red VRS, FKP, MAX; iMAX

Datos de acceso: sin registro

URL: (no operativo; solo Escuela)

\section{Consideraciones finales}

Los servicios de diseminación de datos para procesamiento en tiempo real, así como las correcciones SSR y las efemérides broadcast a través de Cáster, son servicios de gran valor estratégico para actividades de desarrollo e investigación, dado el aumento de la demanda de información georreferenciada.

El uso de estas tecnologías, en la medida que se masifiquen, asegura que los datos georreferenciados a intercambiar estén en el Sistema de Referencia adoptado, aun cuando sean capturados por personas inexpertas (soluciones trasparentes al usuario), ya que el Sistema de Referencia va implícito en las correcciones de los datos de las CORS, al utilizar el método diferencial RTK. Se asegura además una mejora en las coordenadas absolutas de los datos capturados, con respecto a los datos coleccionados por métodos alternativos, en las mismas condiciones.

El uso de posicionamientos con correcciones DGNSS/RTK a través de Ntrip, van a desarrollarse masivamente en la medida que existan CORS suficientes para obtener soluciones precisas, con conectividad GPRS/3G segura a través de Internet. Dependerá en gran medida del alcance de la cobertura de telefonía móvil disponible en cada uno de los países y en la región.

El Proyecto SIRGAS, por intermedio del Grupo SIRGAS-RT, tiene por delante un enorme desafío y una oportunidad invalorable de conseguir implementaciones regionales capaces de brindar correcciones diferenciales en tiempo real a través de Ntrip. Las soluciones de red podrán transcender los límites territoriales y alcanzar altos niveles de seguridad y confiabilidad, lo que dependerá en gran medida del avance en la implementación de redes CORS y del desarrollo de las redes de telefonía móvil u otro sistema alternativo de comunicaciones, futuro. 


\section{Bibliografía}

Briceño, A.; Mass, L.; Rubí, I.; Cioce, V. ; Royero, G.; Bacaicoa, L.; Wildermann, E.; Hoyer, M.; Barrios, M. (2009). "GPS Ntrip observations: A new alternative for precise positioning in Venezuela", Revista Técnica de Ingeniería. Universidad de Zulia, vol. 32, núm. 3, Zulia, Venezuela, pp. 200-209.

Brunini, C.; Sánchez, L. (2008). "Reporte 2007-2008”, Boletín Informativo, núm. 13, Subcomisión 1.3b de la IAG. Grupo de Trabajo de la Comisión de Cartografía del IPGH. Sistema de Referencia Geocéntrico para las Américas (SIRGAS). Montevideo, Uruguay.

Brunini, C.; Sánchez, L.; Mackern, M.V.; Martínez-Díaz, W.A.; Luz, R.T.; Noguera, G.; Pérez-Rodino, R. (2012). "Reporte 2011-2012”, Boletín Informativo, núm. 17, Subcomisión 1.3b de la IAG, Grupo de Trabajo de la Comisión de Cartografía del IPGH, Sistema de Referencia Geocéntrico para las Américas (SIRGAS), Concepción, Chile.

Gao, Y.; Chen, K. (2004). "Performance Analysis of Precise Point Positioning Using ReaL-Time Orbit and Clock Products", Journal of Global Positioning Systems, vol. 3, núm. 1-2, Canadá, pp. 95-100.

Hoyer, M. (2012)."Conceptos básicos del posicionamiento GNSS en Tiempo Real. NRIP y Tópicos Relacionados con el tema", Escuela SIRGAS en Posicionamiento GNSS en Tiempo Real (SIRGAS-RT), Grupo SIRGAS-RT, Reunión Sistema de Referencia Geocéntrico para las Américas (SIRGAS), Concepción, Chile.

Hoyer, M.; Costa, S.; Pérez R., Weber, G.; Da Fonseca, E.; Krueger, C.; Junior, N. (2009). "Ntrip in South America Through the SIRGAS-RT Project", Geodesy for Planet Earth - Scientific Program IAG, Buenos Aires, Argentina.

Hoyer, M.; Cioce, V.; Royero, G.; Márquez, A.; Brito, J. (2010). "Utilización de Ntrip en Venezuela: avances y aplicaciones", Presentación en Reunión SIRGAS, Subcomisión 1.3b de la IAG. Grupo de Trabajo de la Comisión de Cartografía del IPGH, Sistema de Referencia Geocéntrico para las Américas (SIRGAS), Lima, Perú.

Noguera, G.; Pérez Rodino, R. (2012). "Proyecto SIRGAS-RT (Tiempo Real)", Escuela SIRGAS en Posicionamiento GNSS en Tiempo Real (SIRGAS-RT), Grupo SIRGAS-RT, Reunión Sistema de Referencia Geocéntrico para las Américas (SIRGAS), Concepción, Chile.

Noguera,G.; Pérez Rodino, R.; Camisay, M.F. (2013). "Infraestructura para transmisión de datos y servicios GNSS en tiempo real enmarcados en SIRGAS", Informe del Grupo SIRGAS-RT, Sistema de Referencia Geocéntrico para las Américas (SIRGAS), Montevideo, Uruguay.

Pérez Rodino, R.; Suárez Silva, N. (2010). "Servicio de Corrección Diferencial de Posicionamiento Global en Tiempo Real a través de Caster-Ntrip, una herra- 
mienta para el presente y futuro", I Congreso de Infraestructura de Datos Espaciales, Montevideo, Uruguay.

Rizos, C.; Janssen, V.; Roberts, C.; Grinter, T. (2012). Australia "Precise Point Positioning: Is the Era of Differential GNSS Positioning Drawing to an End?", Knowing to manage the territory, protect the environment, evaluate the cultural Heritage, FIG Working Week, FIG, Roma, Italia.

Rovera, H.; Pérez Rodino, R. (2009). "The Uruguayan SIRGAS present and future working in Ntrip", Geodesy for Planet Earth - Scientific Program IAG, Buenos Aires, Argentina.

Suárez Silva, N., (2012). "Red Geodésica Nacional Activa de la República Oriental del Uruguay (REGNA-ROU). Infraestructura al servicio del desarrollo de nuevas capacidades mediante el empleo de Tecnologías de la Información Geográfica (TIG)", Cuaderno de Geografia, vol. 1, Biblioteca Nacional, Montevideo, Uruguay, pp. 23-28.

Stürze. A.; Weber, G. (2012). "Real Time GNSS", Escuela SIRGAS en Posicionamiento GNSS en Tiempo Real (SIRGAS-RT), Grupo SIRGAS-RT, Reunión Sistema de Referencia Geocéntrico para las Américas (SIRGAS), Concepción, Chile.

Weber, G.; Dettmering, D.; Gebhard, H.; Kalafus, R. (2005) "Networked Transport of RTCM via Internet Protocol (Ntrip) - IP Streaming for Real Time GNSS Applications", $18^{\text {th }}$ International Technical Meeting of the Satellite Division. ION GNSS, Long Beach, CA, USA. 